\title{
Oncometabolite succinate promotes angiogenesis by upregulating VEGF expression through GPR91-mediated STAT3 and ERK activation
}

\author{
Xianmin $\mathrm{Mu}^{1, *}$, Ting Zhao ${ }^{1, *}$, Che $\mathrm{Xu}^{1}$, Wei Shi ${ }^{4}$, Biao Geng ${ }^{1}$, Jiajia Shen ${ }^{2}$, Chen \\ Zhang ${ }^{1}$, Jinshun Pan ${ }^{1}$, Jing Yang ${ }^{1}$, Shi Hu${ }^{1}$, Yuanfang Lv ${ }^{1}$, Hao Wen ${ }^{2}$, Qiang You ${ }^{1,3}$ \\ ${ }^{1}$ Department of Biotherapy, Second Affiliated Hospital, Nanjing Medical University, Nanjing, Jiangsu 210011, China \\ ${ }^{2}$ Department of Surgery, Second Affiliated Hospital, Nanjing Medical University, Nanjing, Jiangsu 210011, China \\ ${ }^{3}$ Department of Immunology, Nanjing Medical University, Nanjing, Jiangsu 211166, China \\ ${ }^{4}$ Department of Drug Screening and Evaluation, Chia Tai Tianqing Pharmaceutical Group Co., Ltd, Nanjing, Jiangsu 210023, \\ China \\ *These authors have contributed equally to this work
}

Correspondence to: Qiang You, email: Qiang.You@njmu.edu.cn

Keywords: succinate, GPR91, gastric cancer, angiogenesis, ERK1/2

Received: October 20, $2016 \quad$ Accepted: December 23, $2016 \quad$ Published: January 04, 2017

\section{ABSTRACT}

Altered cellular metabolism is now generally acknowledged as a hallmark of cancer cells, the resultant abnormal oncometabolites cause both metabolic and nonmetabolic dysregulation and potential transformation to malignancy. A subset of cancers have been found to be associated with mutations in succinate dehydrogenase genes which result in the accumulation of succinate. However, the function of succinate in tumorigenesis remains unclear. In the present study, we aim to investigate the role of oncometabolite succinate in tumor angiogenesis. Our data demonstrated the accumulation of markedly elevated succinate in gastric cancer tissues compared with that in paracancerous tissues. Moreover, succinate was able to increase the chemotactic motility, tube-like structure formation and proliferation of primary human umbilical vascular endothelial cells (pHUVECs) in vitro, as well as promoting the blood vessel formation in transgenic zebrafish. Our mechanistic studies reveal that succinate upregulates vascular endothelial growth factor (VEGF) expression by activation of signal transducer and activator of transcription 3 (STAT3) and extracellular regulated kinase (ERK)1/2 via its receptor GPR91 in a HIF-1a independent mechanism. Taken together, these data indicate an important role of the succinate-GPR91 axis in tumor angiogenesis, which may enable development of a novel therapeutic strategy that targets cancer metabolism.

\section{INTRODUCTION}

Dysregulated metabolism is generally accepted as a characteristic hallmark of cancer cells [1] which exhibit altered metabolic phenotype when compared with nonmalignant cells [2]. Mutations in the metabolic genes like fumarate hydratase, succinate dehydrogenase, and isocitrate dehydrogenase lead to the accumulation of fumarate, succinate, and D-2-hydroxyglutarate, respectively [3], which cause both metabolic and nonmetabolic dysregulation and potential transformation to malignancy.

Angiogenesis is another biological hallmark of cancer [1]. The normal vasculature becomes quiescent following the morphogenesis. In contrast, the angiogenic switch during tumorigenesis is almost always activated and remains on, causing quiescent vasculature to sprout new vessels that help sustain expanding neoplastic growths continually [4]. Angiogenesis is a complicated multistep process which contains steps of the migration, proliferation, tube formation, invasion and finally capillary network formation of vascular endothelial cells [5], and is tightly regulated directly or indirectly by the dynamic balance between angiogenic stimulators and inhibitors.

As one of metabolic products in the tumor environment, lactate has been proved to promote cancer angiogenesis [6]. Another oncometabolite succinate was also found to accumulate in certain cancers which mainly caused by mutation of succinate dehydrogenase gene. However, its 
role in tumorigenesis remains unclear. As an intermediate of the tricarboxylic acid cycle, succinate is essential for adenosine triphosphate generation in mitochondria and also a crucial metabolite in several metabolic pathways. It is normally present in the circulation of blood at about $5 \mu \mathrm{M}$ concentration $[7,8]$, while under pathological conditions it is accumulated in extra-cellular spaces. High succinate levels (from $150 \mu \mathrm{M}$ to millimole) have been detected in the urine and plasma or the cerebral white matter of patients with metabolic diseases $[9,10]$. Besides as energy metabolism, recent studies have demonstrated the new role of succinate as a cellular signaling molecule. For example, succinate accumulates in succinate dehydrogenase-deficient tumors like gastrointestinal stromal tumors (GIST), inhibits prolyl hydroxylase activity with subsequent HIF-1 $\alpha$ stabilization and therefore drives cancer progression $[11,12]$. Succinate also enhances interleukin-1 $\beta$ and VEGF production in a HIF-1 $\alpha$-depend pathway [9]. These significant findings indicate that succinate is capable of acting as an intracellular messenger which induces the alterations of gene expression in tumors by targeting HIF-1 $\alpha$. Meanwhile, succinate was demonstrated as an extracellular circulating signaling molecule in a para- and endocrine mode mediated by its specific plasmalemmal succinate receptor [13], which probably beyond HIF-1 $\alpha$ stabilization.

G protein-coupled receptor-91 (GPR91), also known as succinate receptor 1 (SUCNR1) [10], is expressed in several highly vascularized tissues, including retina, heart, liver, kidney and white adipose tissue, and even on the surface of human monocyte-derived dendritic cells. GPR91 activation triggered by local extracellular succinate inhibits lipolysis in white adipose tissue, governs retinal angiogenesis, increases the release of renin in glomerular endothelium, enhances the

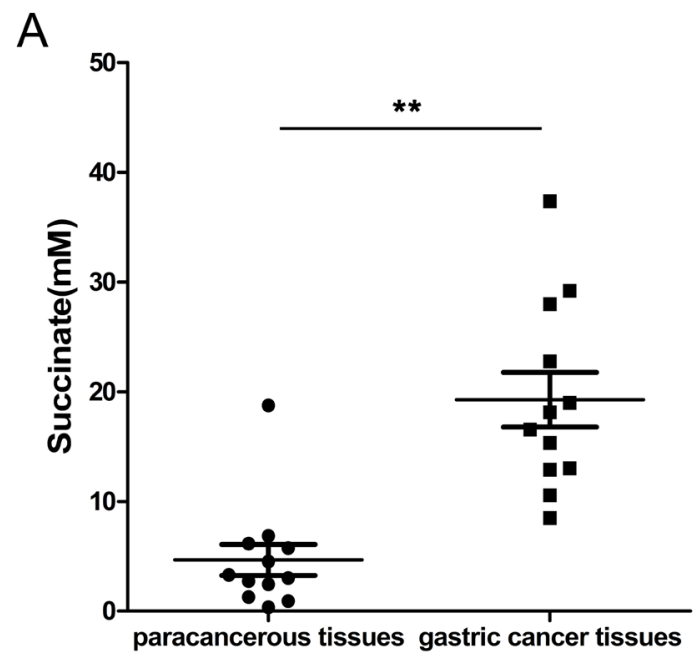

secretion of pro-inflammatory cytokines by dendritic cells for activation of $\mathrm{T}$ helper cells, and also serves as a signaling regulator of hepatic fibrosis [13-20].

The comprehensive roles of succinate and its specific receptor in the development of tumor angiogenesis have not been investigated extensively. In the present study, we examined succinate levels in human gastric cancer tissues and demonstrated that succinate functioned as a biologically active molecule through its receptor GPR91, which bridges a gap between tumor-altered metabolism and angiogenesis.

\section{RESULTS}

\section{Comparison of succinate levels in gastric cancer tissues/paracancerous tissues, cell culture supernatants from normal cells and gastric cancer cells}

Succinate levels were measured in 12 pairs of human gastric adenocarcinoma and tumor-adjacent tissues, which were obtained from stage II-IV male patients with low/ moderate-differentiated gastric adenocarcinoma over 45 years of age. As shown in Figure 1A, the levels of succinate in gastric cancer tissues were significantly higher than that in adjacent normal tissues. To detect the extracellular succinate levels of different cell lines, the cells were cultured in RPMI 1640 media (no phenol red) supplemented with $0.5 \%$ FBS for $24 \mathrm{~h}$ and the supernatants were collected for measurement. Markedly elevated succinate levels in human gastric cancer cells (NCI-N87, BGC-823, AGS and SGC7901) culture were observed compared to human normal gastric epithelial cell (GES-1) culture (Figure 1B). These

B

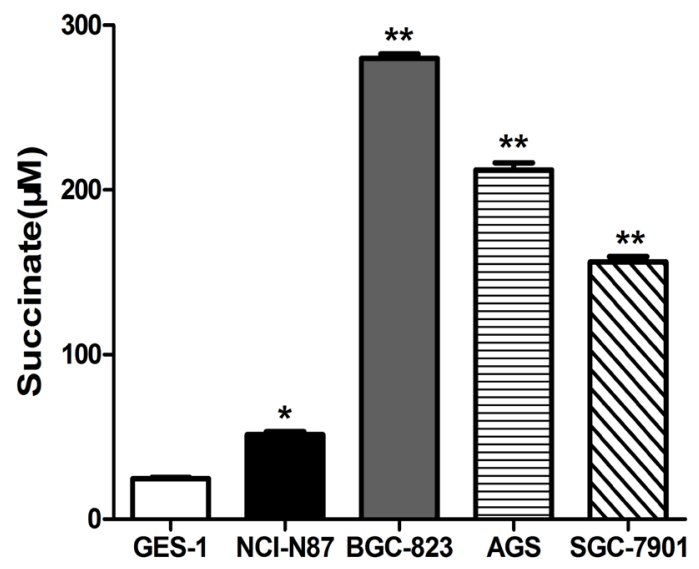

Figure 1: Succinate level is significantly higher in human gastric cancer tissues and cancer cell lines compared with adjacent tissues and normal cell line, respectively. A. Succinate levels were measured in twelve pairs of human gastric adenocarcinoma and tumor-adjacent tissues, which were collected from stage II-IV gastric cancer patients. $* * p<0.01$ compared with adjacent tissues. B. Succinate levels were determined in the culture supernatants of human gastric cancer cells and normal gastric epithelial cells. ${ }^{*} \mathrm{p}<0.05$ and $* * \mathrm{p}<0.01$ versus the culture supernatants of GES-1 cells. 
findings implicate that gastric cancer cells exhibit altered metabolic patterns when compared with nonmalignant cells at least in terms of succinate levels.

\section{Succinate increases the chemotactic motility, capillary structure formation and proliferation of pHUVECs in vitro}

The effect of succinate on pHUVEC proliferation was assessed by BrdU incorporation assay. As a result, treatment with $400 \mu \mathrm{M}$ or $800 \mu \mathrm{M}$ of succinate significantly increased the viability of primary pHUVEC when compared to control (Figure 2A). Since the migration of endothelial cells is a crucial step in angiogenesis and cancer progression [21], the effects of succinate on chemotactic motility of pHUVECs were evaluated by transwell and wound-healing migration assays. As shown in Figure $2 \mathrm{~B}$ and $2 \mathrm{C}$, succinate significantly increased pHUVEC migratory straight distance when compared to PBS control in a concentration-dependent manner. Since the differentiation of endothelial cells and the formation of tube-like structures are essential for angiogenesis, the
A

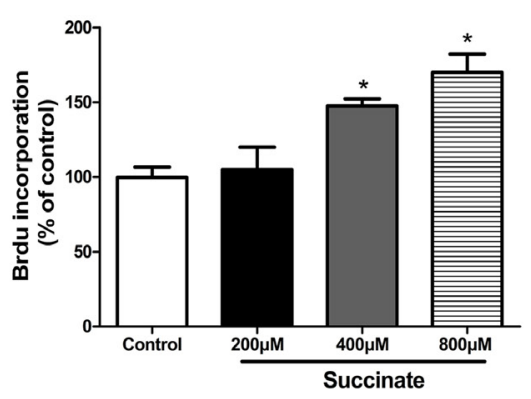

C
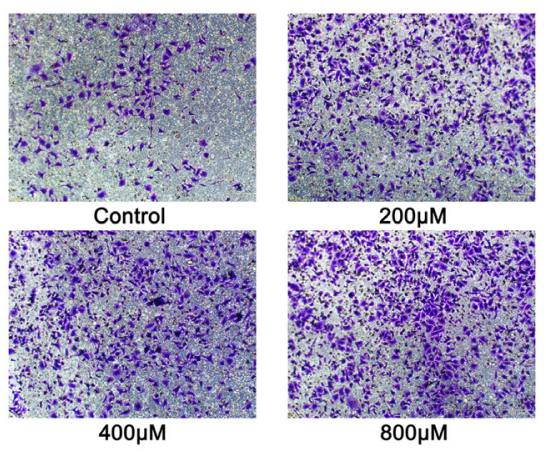

D

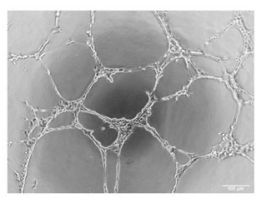

Control

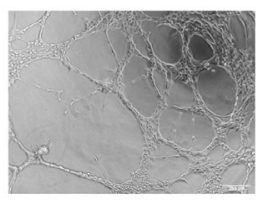

$400 \mu \mathrm{M}$

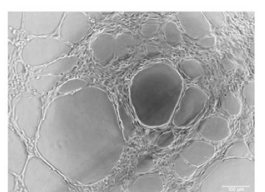

$200 \mu \mathrm{M}$

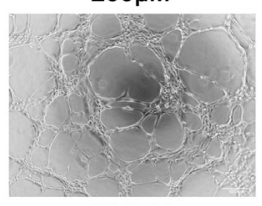

$800 \mu \mathrm{M}$
B
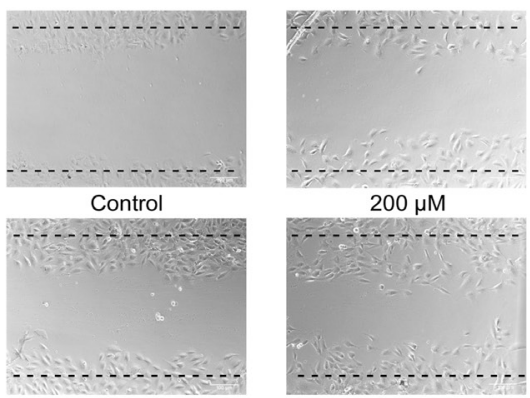

$400 \mu \mathrm{M}$

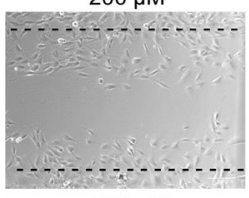

$800 \mu \mathrm{M}$
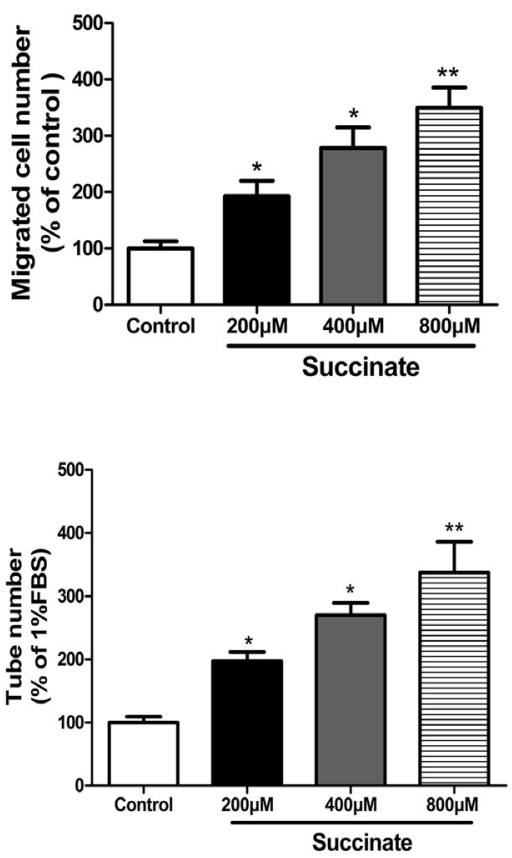

Figure 2: Succinate increases the proliferation, chemotactic motility and capillary structure formation of pHUVECs in vitro. A. BrdU incorporation assay was used to determine the proliferation of pHUVECs in the presence of various concentration of succinate. B. Wound-healing migration assay was applied to observe the effect of succinate on pHUVECs migration. The confluent cells were scratched by pipette and cultured in the presence of various concentration of succinate (magnification, $\times 100$ ). C. Effect of succinate on pHUVECs migration was also evaluated by transwell assay. pHUVECs were seeded in the upper transwell chamber. The bottom chamber was filled with various concentrations of succinate. After $8 \mathrm{~h}$ of co-culture, the migrated cells were quantified by manual counting (Magnification, $\times 100)$. D. Capillarylike formation of pHUVECs was observed in the presence of various concentration of succinate. pHUVECs $\left(2.0 \times 10^{4}\right.$ cells $/$ well $)$ were placed in 96-well plates coated with growth factor reduced matrigel. Six hours later, tubular structures were photographed (Magnification, $\times 100$ ). Three independent experiments (A, B, C and D) data are presented as mean \pm SD. ${ }^{*} \mathrm{p}<0.05$ and ${ }^{* *} \mathrm{p}<0.01$ versus PBS control. 
ability of succinate to promote tube formation was further investigated in a three-dimensional model of endothelial cell capillary tube formation in Matrigel. Stimulation with $10 \mathrm{ng} / \mathrm{mL}$ VEGF promoted pHUVECs differentiating and forming robust tubular-like structures (data not shown). Similarly, succinate enhanced the formation of a tube-like network as well after $6 \mathrm{~h}$ of culture as shown in Figure 2D. Quantitative measurements confirmed that succinate triggered a significant increase in total tubes.

\section{Succinate promoted the vessel formation in zebrafish embryo}

To study the effect of succinate on angiogenesis in vivo, transgenic (flk1: GFP) zebrafish embryos, which express GFP in all vascular endothelial cells, were used. The development of zebrafish SIVs was visualized using fluorescence stereomicroscope. SIVs length was measured with Image Pro Plus. The representative photos and quantitative analysis of zebrafish SIVs were shown in Figure 3. The small molecule kinase inhibitor PTK787 was used in pro-angiogenesis in vivo model, which inhibits human VEGFRs and therefore effectively blocks angiogenesis [22]. As shown in Figure 3A, treatment with $0.2 \mu \mathrm{g} / \mathrm{mL}$ PTK787 prevented the formation of blood vessels. As anticipated, the simultaneous treatment of $\mathrm{VEGF}_{165}$ significantly restored angiogenesis. In line with the observations described above, the length of SIVs was significantly increased in zebrafish embryos treated with succinate at the concentration above $100 \mu \mathrm{M}$ when compared to PBS control, which indicates a proangiogenic effect of succinate on zebrafish embryo in a dose-dependent manner.

\section{Succinate activates STAT3 and ERK signaling through GPR91}

In order to investigate the pro-angiogenic mechanism of succinate, we first determined the timing and dosage of succinate treatment on STAT3 and ERK activation. pHUVECs were cultured in the presence of $400 \mu \mathrm{M}$ succinate and measured ERK1/2 and STAT3 expression at different time point $(5,15,30$ and $60 \mathrm{~min})$. As shown in Figure 4A, the peak of pERK1/2 expression level is at $15 \mathrm{~min}$, while the peak of pSTAT3 expression is at $30 \mathrm{~min}$. The data suggest ERK activation by succinate
A

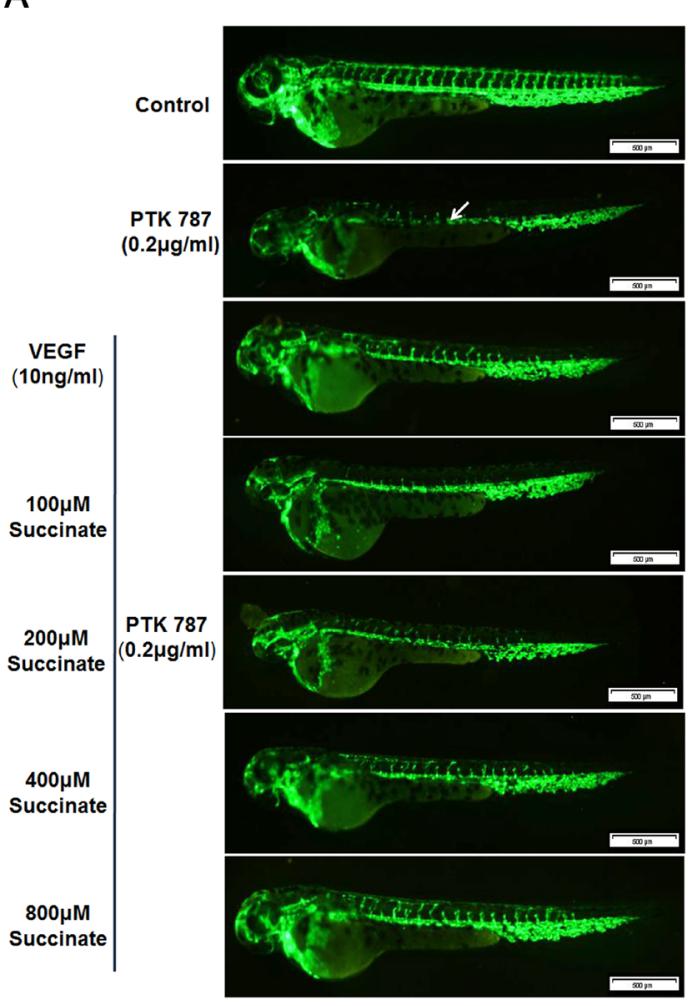

B
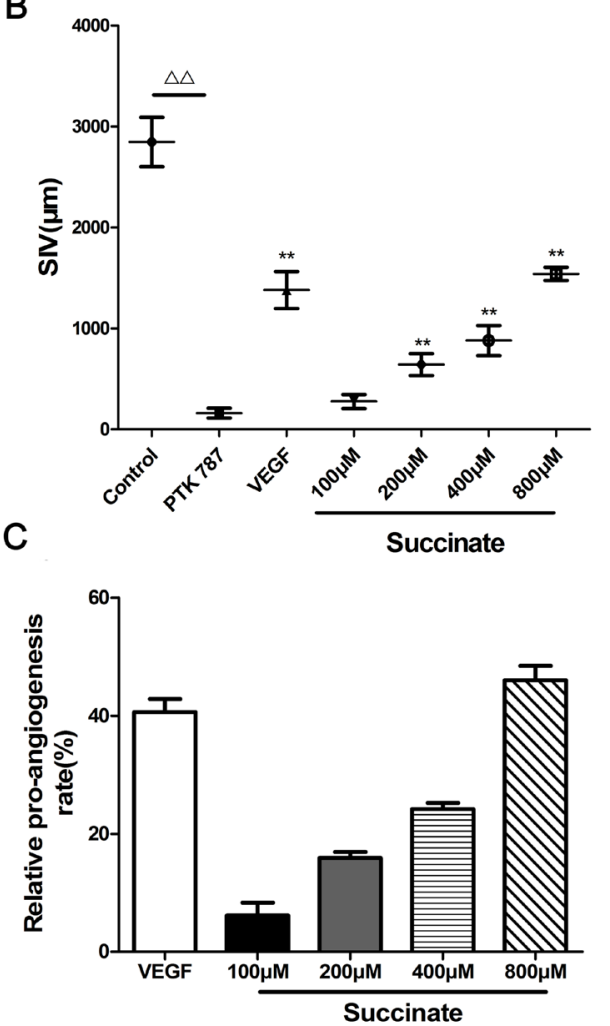

Figure 3: Succinate promotes vessel formation in the zebrafish embryo. A. Effect of succinate on angiogenesis was evaluated in zebrafish embryos. Transgenic (flk1: GFP) zebrafish embryos were picked out and distributed into a 24-well microplate (9 embryos/ well), and pre-treated with $0.2 \mu \mathrm{g} / \mathrm{mL}$ PTK787 (VEGFR inhibitor, Sigma). Then, various concentration of succinate (0, 100, 200, 400, $800 \mu \mathrm{M})$ or $10 \mathrm{ng} / \mathrm{mL}$ of $\mathrm{VEGF}_{165}$ were added to the culture for twenty-four hours. Representative photographs are listed to show the subintestinal vessels (SIVs) indicated by white arrow. B, C. Quantitative analysis of the overall length of zebrafish SIVs are shown. Data are presented as mean $\pm \mathrm{SD} . * \mathrm{p}<0.05$ and $* * \mathrm{p}<0.01$ versus $\mathrm{PBS}$ control. 
A
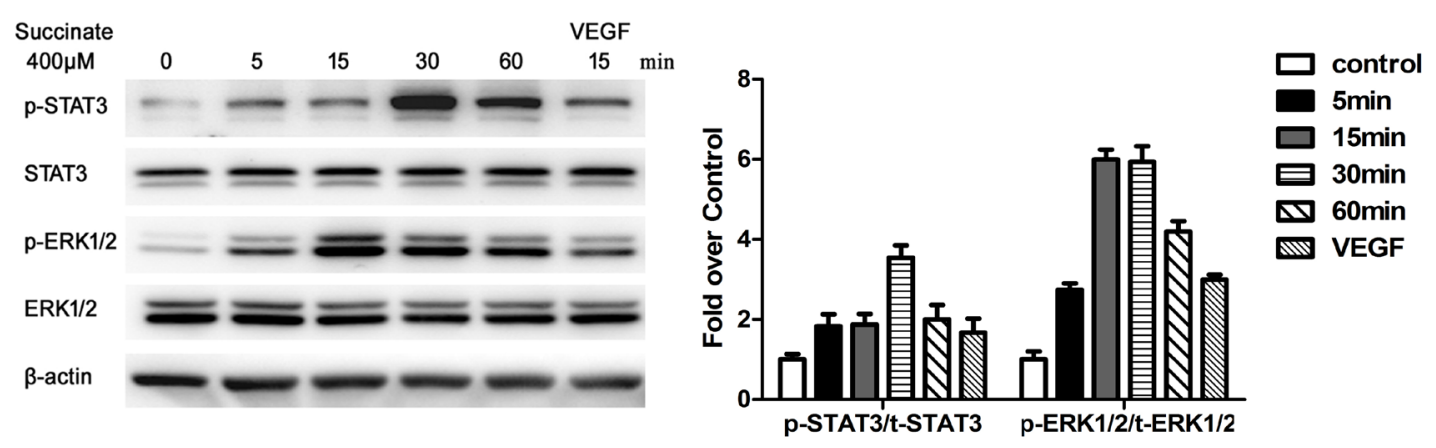

B

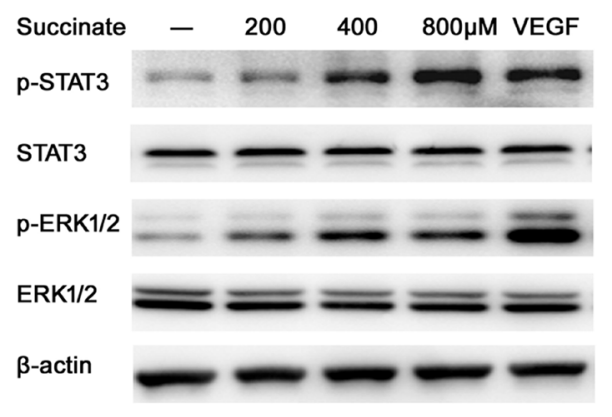

$\mathrm{C}$
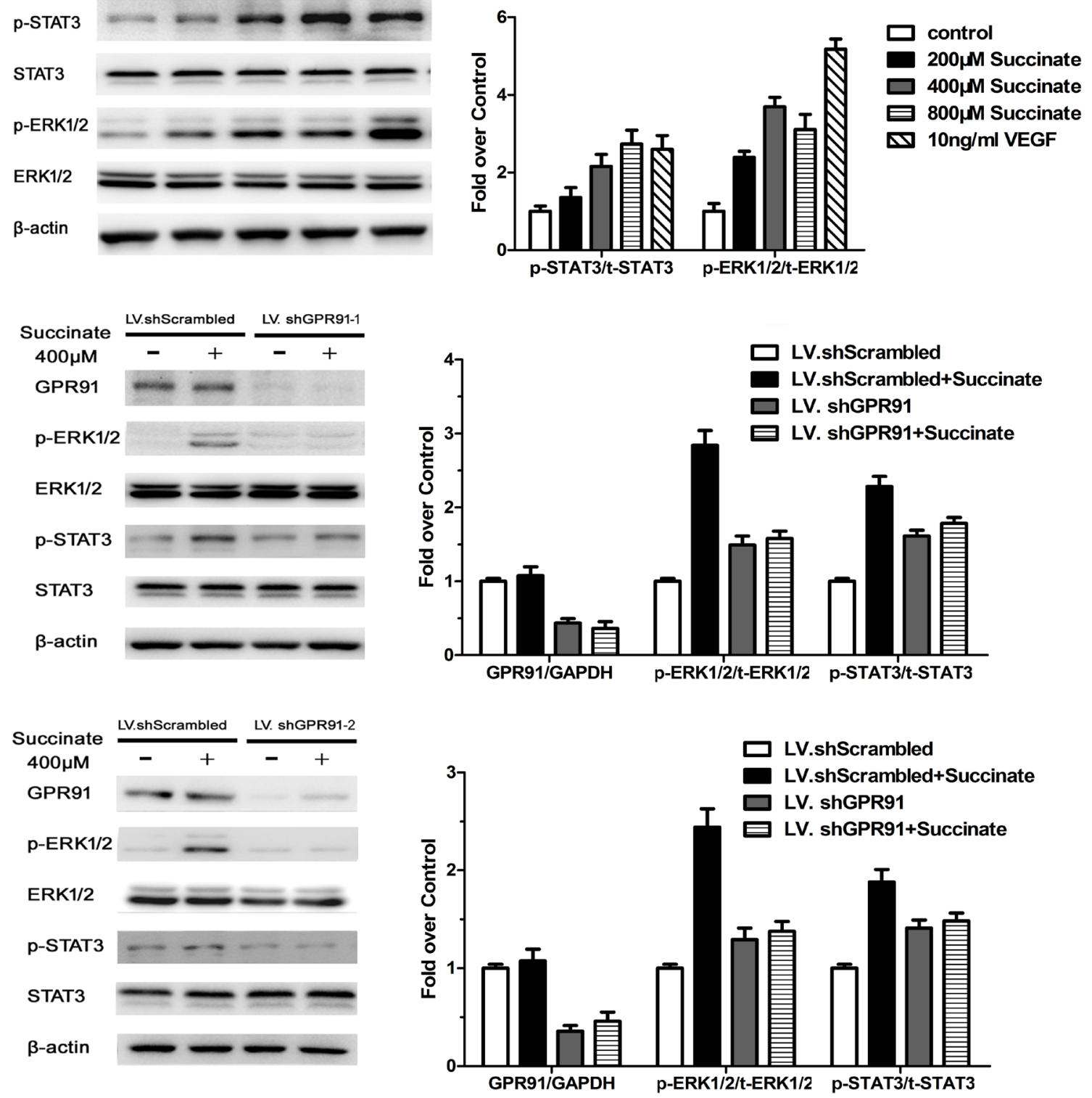

Figure 4: Succinate activates ERK1/2 and STAT3 signaling through GPR91 in pHUVECs. A. STAT3 and ERK1/2 activation were measured by Western blotting in pHUVECs treated with $400 \mu \mathrm{M}$ succinate for different periods of time. B. STAT3 and ERK1/2 activation were determined in pHUVECs cultured in the presence of various concentrations of succinate or $10 \mathrm{ng} / \mathrm{mL}$ of VEGF 165 for 15 min. C. STAT3 and ERK1/2 activation were evaluated in pHUVECs transduced with LV.shScrambled or LV. shGPR91-1, or LV. shGPR91-2 in the presence of succinate for $15 \mathrm{~min}$. The expression of total STAT3 and Erk1/2 protein were also detected. $\beta$-actin was used as loading control. Data were represented as mean $\pm \mathrm{SD} ; \mathrm{n}=3 .{ }^{*} P<0.05$ versus $\mathrm{PBS}$ control. 
is earlier than STAT3. A dose-dependent trend of the activation was further observed when pHUVECs were treated with various concentrations of succinate (Figure 4B). To verify that succinate activated ERK1/2 and STAT3 signaling through the receptor GPR91, we knocked down its expression with two kinds of lentiviruses containing the different shRNA sequences (LV. shGPR91-1 and 2), which were also adopted to exclude the off-target effect. Both LV. shGPR91 (transfection rate of $\sim 95 \%$ ) diminished GPR91 mRNA approximately $85-90 \%$ and the protein expression about 65\%, whereas LV.shScrambled (control) was ineffective to reduce GPR91 expression (Figure 4C). Using this knockdown technique, we observed that the increase in the phosphorylation levels of ERK1/2 and STAT3 was significantly blocked by both LV. shGPR91 $(\mathrm{P}<0.01)$ as shown in Figure $4 \mathrm{C}$. These results indicate that GPR91 mediates the activation of ERK1/2 and STAT3 signaling by succinate.

\section{Succinate promotes angiogenesis by activating ERK1/2 signaling via GPR91 in pHUVECs}

A series of assays including BrdU incorporation, wound-healing migration, transwell and capillarylike formation were applied to evaluate the proangiogenic effect of succinate. pHUVECs transduced with LV.shGPR91 or LV.shScrambled were pre-treated with $10 \mu \mathrm{M}$ ERK1/2 inhibitor selumetinib to assess the activation of succinate on ERK1/2 through GPR91. As shown in Figure $5 \mathrm{~A}-5 \mathrm{D}$, treatment with $400 \mu \mathrm{M}$ of succinate significantly increased the viability, chemotactic motility and capillary structure formation ability of
A

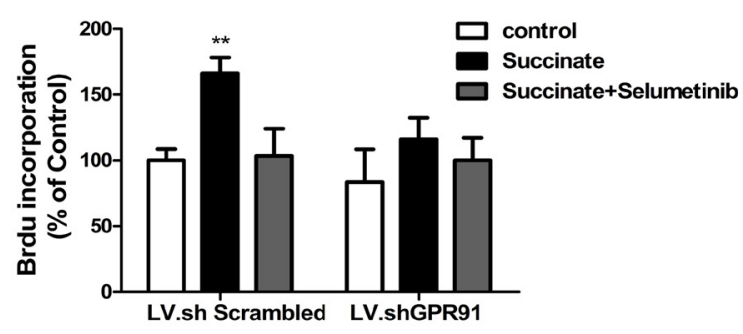

B

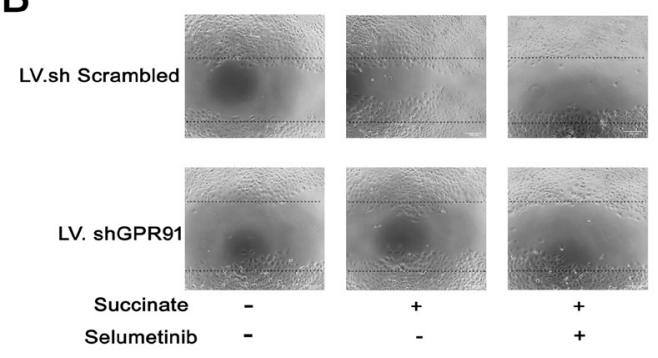

C
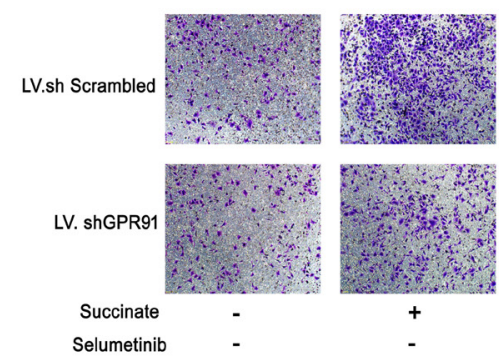

D
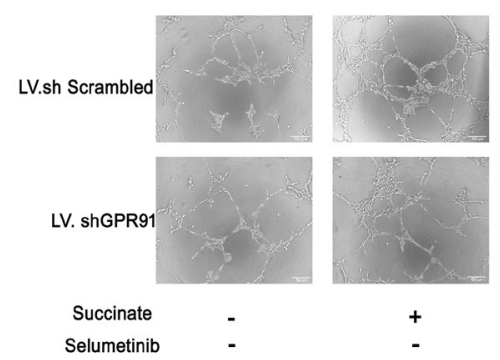
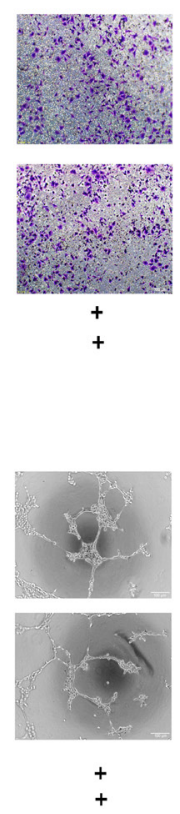

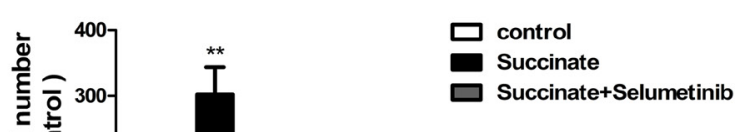

Succinate

$\square$ Succinate+Selumetinib

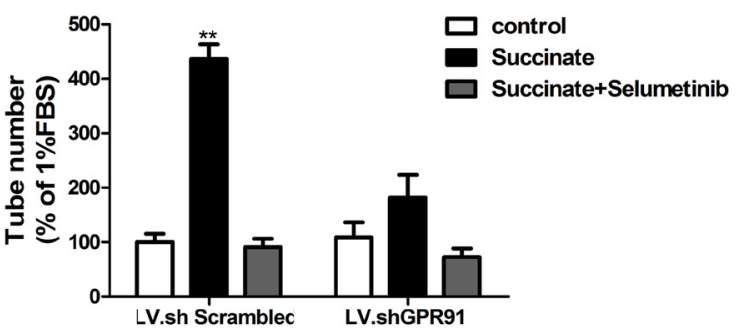

Figure 5: Succinate promotes angiogenesis by activating ERK1/2 signaling through GPR91. pHUVECs, transduced with LV.shGPR91 or LV.shScrambled, were pre-treated with $10 \mu \mathrm{M}$ ERK1/2 inhibitor selumetinib and then cultured in the presence of succinate to evaluate its pro-angiogenic effect. A. BrdU incorporation assay was used to determine the effect of succinate on the proliferation of pHUVECs. B. The wound-healing assay was applied to observe the effect of succinate on pHUVECs migration (magnification, $\times 100)$. C. Transwell assay was also adopted to determine the effect of succinate on pHUVECs migration (Magnification, $\times 100$ ). D. Capillary-like formation of pHUVECs was observed in the presence of succinate (Magnification, $\times 100$ ). The data from three independent experiments are presented as mean \pm SD. ${ }^{*} \mathrm{p}<0.05$ and $* * \mathrm{p}<0.01$ versus LV.shScrambled group. 
pHUVEC compared to the control. However, addition with selumetinib diminished these effects. Interestingly, the pro-angiogenic effect of succinate on pHUVECs was significantly blocked in the cells transduced with LV. shGPR91 $(\mathrm{p}<0.01$, Figure 5A-5D). These data suggest that the activation of ERK $1 / 2$ signaling mediated by GPR91 is important for succinate to promote angiogenesis in pHUVECs.

\section{VEGF upregulation induced by succinate depends on the activation of STAT3 and ERK1/2 signaling in pHUVECs}

Next, we sought to investigate the mechanism of pro-angiogenic function of succinate on pHUVECs. VEGF is a well-known angiogenic mediator that can promote endothelial cells growth. As shown in Figure 6A, $6 \mathrm{~B}$ and $6 \mathrm{C}$, succinate treatment significantly increased VEGF mRNA and protein levels in pHUVECs compared with PBS control group. Since VEGF is the target gene of HIF-1 $\alpha$ transcription factor, we knocked down HIF- $1 \alpha$ gene and determined whether HIF- $1 \alpha$ played a functional role in the effect of succinate. Interestingly, depletion of HIF-1 $\alpha$ didn't affect the upregulation of VEGF induced by succinate as shown in Figure 6A. Instead, ERK inhibitor Selumetinib or STAT3 inhibitor Stattic alone was able to decrease VEGF expression induced by succinate, and the combinations further reduced to the control level (Figure $6 \mathrm{~B}$ and $6 \mathrm{C}$ ). Then, we investigated the role of GPR91 in the regulation of VEGF secretion by succinate. GPR91 knockdown reduced VEGF mRNA by approximately $55 \%$ (Figure 6B) and significantly decreased VEGF protein expression by approximately 34\% (Figure 6C). These data indicated that effect of succinate on VEGF upregulation was through modulating STAT3 and ERK1/2 signaling via GPR91 in pHUVECs.

\section{DISCUSSION}

Altered cellular metabolism is now known as a hallmark of cancer cells. The involved metabolic pathways include aerobic glycolysis, glutamine catabolism, anaplerosis, de novo synthesis of fatty acids and lipids [23]. The resultant abnormally accumulated metabolites cause both metabolic and nonmetabolic dysregulation and potential transformation to malignancy, and herein termed oncometabolites including fumarate, succinate, and D-2-hydroxyglutarate [24]. An increasing body of evidence suggests that the abnormal accumulation of these metabolites can re-edit tumor microenvironment, therefore drive cancer progression.

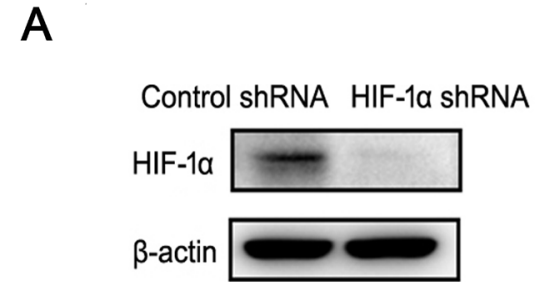

B

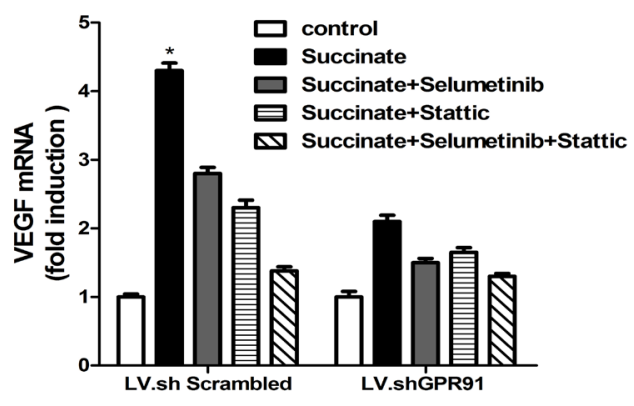

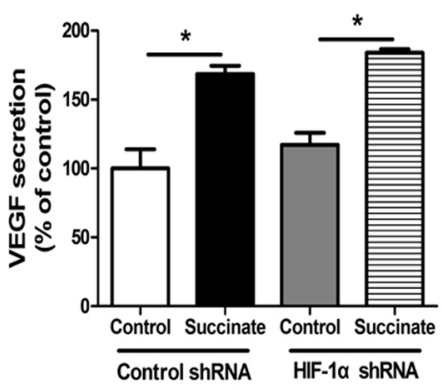

C

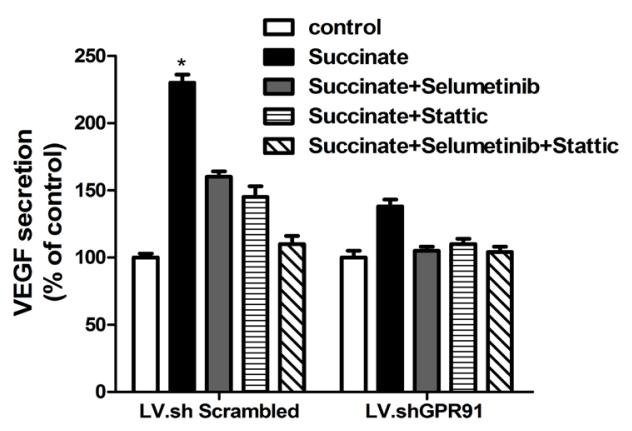

Figure 6: Succinate enhances VEGF expression by activating ERK1/2 and STAT3 signaling through GPR91. A. Succinate increases VEGF expression independent of HIF-1 $\alpha$ gene. The expression of HIF-1 $\alpha$ protein in LV.shScrambled-transduced or LV.shHIF$1 \alpha$-transduced AGS cells was determined by Western blotting (left). The cell culture supernatant was collected from the transduced cells in the presence of $400 \mu \mathrm{M}$ succinate for $24 \mathrm{~h}$ and measured VEGF level (right). VEGF mRNA levels B. or protein concentration in the supernatant $\mathbf{C}$. were determined by RT-qPCR or ELISA in pHUVECs cultured in the presence of succinate for $12 \mathrm{~h}$, which were pre-treated with $10 \mu \mathrm{M}$ Selumetinib (ERK1/2 inhibitor) or/and 10 $\mu \mathrm{M}$ Stattic (STAT3 inhibitor) for ten minutes. Each column represents the mean \pm SD $(\mathrm{n}=3) .{ }^{*} \mathrm{P}<0.05$ versus PBS control. 
Succinate, a crucial intermediate metabolite in several metabolic pathways, is accumulated in extracellular spaces under pathological condition, for instance, hyperglycemic or hypoxia. High concentrations of succinate (from $150 \mu \mathrm{M}$ to millimole) have been detected in the urine and plasma or the cerebral white matter of patients with metabolic diseases [9, 10]. Dysfunction of the succinate dehydrogenase, a heterotetrameric enzyme complex participating in both the citric acid cycle and electron transport chain, leads to succinate accumulation in human tumors such as papillary thyroid cancers, thyroid C-cells hyperplasia, pancreatic neuroendocrine tumors, paragangliomas, chronic lymphocytic leukemia, renal carcinomas and pituitary adenomas [7, 25]. However, there is no evidence to show whether oncometabolite succinate elevation is present in gastric cancer. Using tissue samples from patients with stage II-IV gastric cancer we demonstrated that succinate levels were increased in human gastric adenocarcinoma compared with tumor-adjacent tissues (Figure 1A). In addition, our data reveal that human gastric cancer cells (NCI-N87, BGC-823, AGS and SGC-7901) produce more succinate than normal epithelial GES-1 cells (Figure 1B).

Angiogenesis inducing is another hallmark of cancer $[1,4]$. It has been shown that succinate accumulates in the hypoxic retina of rodents as a potent mediator of vessel growth in the settings of both normal retinal development and proliferative ischemic retinopathy [15]. Therefore, we focus on investigating the function of succinate in tumor angiogenesis. Herein, we examined pro-angiogenic effects of succinate in vitro and in vivo. As shown in Figure 2 and 3, succinate was able to increase chemotactic motility, tube-like structure formation and proliferation of primary human umbilical vascular endothelial cells (pHUVECs), as well as promoting blood vessel formation in transgenic zebrafish.

The well-known prototype of angiogenesis inducers is VEGF, which is a well-documented angiogenic mediator that can promote endothelial cells growth, maturation and survival [26]. In the present study, we demonstrate that succinate mediates HIF-independent upregulation of VEGF and promote tumor angiogenesis via plasmalemmal GPR91 at $\mathrm{pH}$ 7.4. Succinate has been identified as an inhibitor of prolyl 4-hydroxylases (P4Hs) in vitro to upregulate HIF-1 $\alpha$ expression, which has Ki values of 350$460 \mu \mathrm{M}[11]$. It doesn't penetrate cell membrane easily and has limited uptake into cells, especially in PH7.4 [27, 28]. Therefore, we administered certain amount of succinate which didn't induce HIF-1 $\alpha$ stabilization. Interestingly, VEGF expression induced by succinate is through GPR91 receptor in HIF-1 $\alpha$-independent manner (Figure $6 \mathrm{~A})$. It is noteworthy that the effective concentration of succinate for a half-maximal (EC50) response on GPR91 is $28-56 \mu \mathrm{M}[10]$, higher than the $10 \mu \mathrm{M}$ succinate used in aortic rings assay which resulted in minimal sprouting [15], indicating that GPR91 would be activated at higher concentrations of succinate in pHUVECs. We find that $200 \mu \mathrm{M}$ succinate already can stimulate VEGF production and promote tumor angiogenesis beyond HIF-1 $\alpha$.

MEK-ERK signaling pathway plays a central role in tumor angiogenesis [29]. Its activation promotes sprouting and increases vessel length in tumors [30]. Succinate has been shown to activate ERK1/2 through Gq and $\mathrm{Gi}$ [31]. In the present study, we demonstrated that succinate promoted angiogenesis by ERK1/2 signaling via GPR91 in pHUVECs. Interestingly, succinate also activated STAT3 tyrosine 705 phosphorylation (Figure 3). STAT3 is known as a direct transcriptional activator of VEGF gene by its phosphorylation at tyrosine 705, which is essential for its dimer formation, nuclear translocation, and DNA binding activity [32]. Transactivation of the STAT3 C-terminal domain depends on its tyrosine 705 and serine 727 . ERK1/2 specifically phosphorylates STAT3 at serine 727 , which negatively modulates STAT3 tyrosine phosphorylation [33]. We presume that ERK1/2 activation would reduce STAT3 tyrosine 705 phosphorylation at a later time in pHUVECs since the phosphorylation of STAT3 tyrosine 705 was not be detected at $24 \mathrm{~h}$ after succinate stimulation, instead ERK1/2 signaling activity was still upregulated (data not shown). These data suggest that STAT3 activation participates in the early stage of succinate induced pro-angiogenesis, whereas sustained activation of ERK1/2 via GPR91 mainly accounts for the angiogenic function of succinate in the tumor microenvironment.

Angiogenesis is required for invasive tumor growth and metastasis, and plays an essential role in the control of cancer progression. Our data demonstrate the proangiogenic role of oncometabolite succinate, which can upregulate VEGF expression by activating ERK1/2 and STAT3 signaling via GPR91 receptor. These findings have potential clinical implications that targeting succinateGPR91 axis may be applied as a novel therapeutic strategy to inhibit tumor angiogenesis.

\section{MATERIALS AND METHODS}

\section{Human tissues}

Clinical samples from 12 patients with histological proven low/ moderate-differentiated gastric adenocarcinoma were obtained at the Second Affiliated Hospital of Nanjing Medical University between May 2014 and November 2014. Both tumor tissues and non-cancerous tissues were confirmed histologically. This study was approved by the ethics committee of the Second Affiliated Hospital of Nanjing Medical University (2016 KY-043).

\section{Reagents and antibodies}

Rabbit anti-human p-ERK1/2 monoclonal antibody, rabbit anti-human p-STAT3 (705) monoclonal 
antibody, rabbit anti-human STAT3 monoclonal antibody, rabbit anti-human $\beta$-actin monoclonal antibody and rabbit anti-human ERK1/2 monoclonal antibody were purchased from Cell Signaling Technology (Boston, MA, USA). Rabbit anti-human GPR91 polyclonal antibody were purchased from Novus Biologicals LLC (Littleton, CO, USA). Secondary antibodies were obtained from Invitrogen (Carlsbad, CA, USA). Growth factor-reduced Matrigel was purchased from BD Biosciences (Bedford, MA, USA), and recombinant human $\mathrm{VEGF}_{165}$ was obtained from R\&D Systems (Minneapolis, MN, USA). Endothelial cell medium (ECM, 1001) was purchased from ScienCell (Carlsbad, CA, USA). Succinate was obtained from Sigma. Selumetinib and Stattic were from Selleck Chemicals (Houston, TX, USA).

\section{Cell lines and cell culture}

Primary human umbilical vascular endothelial cells (pHUVECs) were isolated from human umbilical cord veins by collagenase (Sigma) digestion as described previously [34]. Briefly, pHUVECs were maintained in endothelial cell medium at $37^{\circ} \mathrm{C}$ under a humidified 95\%:5\% (v/v) mixture of air and $\mathrm{CO}_{2}$. Ten $\mu \mathrm{M}$ of Selumetinib or Stattic was applied to inhibit ERK1/2 or STAT3 activation.

Human gastric mucosal epithelial cell line GES1 and human gastric cancer cell lines AGS (lowdifferentiated human gastric adenocarcinoma), NCI-N87 (well-differentiated human carcinoma), BGC-823 (low-differentiated human gastric adenocarcinoma) and SGC-7901 (moderate-differentiated human gastric adenocarcinoma) were cultured in RPMI 1640 medium supplemented with $10 \% \mathrm{FBS}, 100$ units/mL penicillin, and $100 \mu \mathrm{g} / \mathrm{mL}$ streptomycin. NCI-N87 and AGS cells were from the American Type Culture Collection. GES-1, BGC823 and SGC-7901 cells were obtained from the Shanghai Institutes for Biological Sciences, Chinese Academy of Sciences, China.

\section{Lentiviruses and endothelial cells infection}

Lentiviruses carrying shRNA sequence targeting human GPR91 or human HIF-1 $\alpha$ were obtained from GeneChem (Shanghai, China). The viruses were used to infect cells in the presence of Polybrene. Forty-eight hours later, pHUVECs were cultured in medium containing puromycin for cells screening. The clones stably knocking down GPR91 were identified and verified by Western blotting and qPCR. The Short-hairpin RNA sequences are listed: shGPR91-1: GCCTCTCAACTTGGTCATCATctcg agATGATGACCAAGTTGAGAGGC; shGPR91-2: CCTT AACTCATAGACATCAATctcgagATTGATGTCTATGAG TTAAGG; shHIF-1 $\alpha-1$ : CAGCTGACCAGTTATGATTG TctcgagACAATCATAACTGGTCAGCTG; shHIF-1 $\alpha-2$ : AACTAACTGGACACAGTGTGTctcgagACACACTGT
GTCCAGTTAGTT. The non-targeting control sequence is 5'-TTCTCCGAACGTGTCACGT-3'.

\section{Succinate level measurement}

Succinate level was determined by Succinate Colorimetric Assay Kit (Sigma-Aldrich Inc., St Louis, MO, USA) according to the manufacturer's instructions. Briefly, tissues $(50 \mathrm{mg})$ were rapidly homogenized on ice in $500 \mu \mathrm{L}$ of ice-cold Succinate Assay Buffer and centrifuged at $10,000 \times \mathrm{g}$ for 5 minutes to remove insoluble material. For cell cultures, the supernatants of cells $\left(1 \times 10^{6}\right.$ cells $)$ were collected and diluted $1: 1$ with $0.5 \mathrm{M}$ Tris- $\mathrm{HCl}$ ( $\mathrm{pH} 8.0$ ). Then, the samples were added into a 96-well plate in duplicate wells and mixed with the appropriate Reaction Mix. The resultant mixtures were further incubated at $37^{\circ} \mathrm{C}$ for 30 minutes. The succinate concentration was determined by the standard curve using spectroscopy at $450 \mathrm{~nm}$ wavelength, and each measurement was performed in triplicate.

\section{Cell proliferation assay by bromodeoxyuridine (BrdU) incorporation}

Cell proliferation was evaluated by a BrdU assay kit (Roche Applied Science, Mannheim, Germany) according to the manufacturer's instructions. Briefly, pHUVECs $\left(5 \times 10^{3}\right.$ cells/well) were seeded in 96 -well plates overnight. Then, the cells were starved in serum-free ECM for $6 \mathrm{~h}$ and further cultured in $100 \mu \mathrm{L}$ of serum-free ECM containing various concentrations of succinate for $24 \mathrm{~h}$. Ten $\mu \mathrm{L}$ of a BrdU-labeling solution was added to each well for $4 \mathrm{~h}$ and the wells were dried, fixed, and measured the optical density at a wavelength of $450 \mathrm{~nm}$. These assays were repeated in triplicate.

\section{Wound-healing migration assay}

pHUVECs were seeded into 6-well plates pre-coated with $0.1 \%$ gelatin (Sigma) to grow to full confluence and subsequently starved in serum-free ECM for $6 \mathrm{~h}$ to synchronization. The cells were wounded with pipette tips and cultured in serum-free ECM with or without various concentrations of succinate. Images were captured (Olympus; magnification, $\times 100$ ) after $12 \mathrm{~h}$ of incubation at $37^{\circ} \mathrm{C}$ in a $95 \%: 5 \%(\mathrm{v} / \mathrm{v})$ mixture of air and $\mathrm{CO}_{2}$.

\section{Transwell migration assay}

The chemotactic motility of pHUVECs was determined using a Transwell migration assay as described previously [34]. Briefly, the upper chambers were seeded with pHUVECs $\left(1.8 \times 10^{4}\right.$ cells $)$ suspended in $200 \mu \mathrm{L}$ of serum-free ECGM; the bottom chambers were filled with $600 \mu \mathrm{L}$ of serum-free ECGM supplemented with various concentrations of succinate. After $8 \mathrm{~h}$ of culture, the nonmigrated cells were removed, and the migrated cells were 
fixed and stained with $1 \%$ crystal violet. Images were photographed (Olympus; magnification, $\times 100$ ) and the migrated cells were counted manually.

\section{Capillary-like tube formation assay}

Tube formation was assessed as previously described [34]. Growth factor-reduced Matrigel was pipetted into pre-chilled 96 -well plates $(60 \mu \mathrm{L}$ per well $)$ and polymerized for 1 hour at $37^{\circ} \mathrm{C}$. pHUVECs $\left(2.0 \times 10^{4}\right.$ cells/well) were first incubated in serum-free ECM for $6 \mathrm{~h}$, subsequently seeded onto the Matrigel layer in the presence of various concentrations of succinate for $6 \mathrm{~h}$ in 96-well plates. The tubular structures of the endothelial cells were recorded using an inverted microscope (Olympus; magnification, $\times 100$ ). The number of meshes per field was calculated randomly from five fields.

\section{Zebrafish embryos angiogenesis assay}

Transgenic (flk1: GFP) zebrafish embryos were generated by natural pair-wise mating, collected and maintained in $28.5^{\circ} \mathrm{C}$ incubator as previously described [35]. Healthy, limpid, and fluorescent embryos were picked out at 24 hours post-fertilization (hpf) and distributed into a 24-well microplate (9 embryos/well) containing Holt Buffer. A final concentration of $0.2 \mu \mathrm{g} /$ mL PTK787 (VEGFR tyrosine kinase inhibitor, Sigma) was added to each well at 24 hours post-fertilization (hpf) and washed out at $30 \mathrm{hpf}$. Meanwhile, various concentration of succinate $(100,200,400$ and $800 \mu \mathrm{M})$ or $10 \mathrm{ng} / \mathrm{mL}$ of $\mathrm{VEGF}_{165}$ were added to the culture media. PBS served as the control. Embryos were maintained in a $28.5^{\circ} \mathrm{C}$ incubator for an additional $24 \mathrm{~h}$, placed onto a glass slide, and photographed using fluorescence stereomicroscope. The overall length of the subintestinal vessels (SIVs) were quantified using Image J software. Pro-angiogenic effects were defined as the increase of SIVs length. The experimental protocol was established according to institutional ethical guidelines for animal experiments and approved by the Ethics Committee of the Second Affiliated Hospital of Nanjing Medical University.

\section{Quantitative real-time PCR for mRNA expression analyses}

Total RNA was extracted from pHUVECs treated with succinate or inhibitors for $12 \mathrm{~h}$. For firststrand cDNA synthesis, $500 \mathrm{ng}$ of total RNA was retrotranscribed using Prime Script RT reagent Kit (Thermo Fisher Scientific, Waltham, MA, USA). The resultant cDNA was subsequently amplified with the Maxima SYBR-Green/Rox qPCR Master Mix 2X kit (Thermo Fisher Scientific, Waltham, MA, USA) using the StepOnePlus Real-Time PCR System (Thermo Fisher Scientific). Changes in the expression of VEGF gene was determined relative to the mean critical threshold (CT) values of GAPDH gene. Human VEGF gene primers are 5'-AGGGCAGAATCATCACGAAGT- 3 ' and 5'-AGGGTCTCGATTGGATGGCA-3'. Human GAPDH gene primers are 5'- TTGCCATCAATGACCCCTTCA- 3' and 5'- CGCCCCACTTGATTTTGGA- 3'.

\section{Western immunoblot analysis}

To study the molecular mechanism of succinateinducing angiogenesis, pHUVECs were first starved in serum-free ECM for $6 \mathrm{~h}$ and then treated with or without various concentration of succinate for $15 \mathrm{~min}$ (for GPR91 activation) or $400 \mu \mathrm{M}$ succinate at different timepoints. After stimulation, the cells were harvested and lysed followed by centrifugation. The total protein concentration was determined by Bicinchoninic acid assay. An equal amount of protein from each lysate $(30-40 \mu \mathrm{g})$ was electrophoresed on $8-12 \%$ SDS polyacrylamide gels and probed with specific primary antibodies followed by horseradish peroxidaseconjugated goat anti-rabbit antibody.

\section{Enzyme-linked immunosorbent assay (ELISA)}

The cell culture supernatant was collected from nontransduced, LV.shScrambled-transduced or LV.shGPR91transduced cells treated with different concentration of succinate. Then, VEGF level was measured by VEGF enzyme-linked immunoassay kits (R\&D Systems, Minneapolis, MN, USA) according to the manufacturer's instructions. Each sample was tested in triplicate.

\section{Statistical analysis}

All data reported herein represent means \pm SD from at least three independent experiments. Statistical comparisons between the treated groups and the control group were performed by one-way analysis of variance (ANOVA) followed by Dunnet's test, the difference between two groups was analyzed by Student's t-test. A P value of $<0.05$ was considered statistically significant.

\section{Abbreviations}

pHUVECs, primary human umbilical vascular endothelial cells; HIF, hypoxia-inducible factor; ERK, extracellular regulated kinase; STAT3, Signal transducer and activator of transcription 3; GPR91, G proteincoupled receptor-91; VEGF, vascular endothelial growth factor; MAPK, mitogen-activated protein kinases; ECM, endothelial cell medium; BrdU, bromodeoxyuridine.

\section{CONFLICTS OF INTEREST}

The authors have no conflicts of interest. 


\section{GRANT SUPPORT}

National Natural Science Foundation of China 81471543,81671543 (to Q.Y.), National Natural Science Foundation of China 81402204 (to X.M.), Jiangsu Provincial Natural Science Foundation BK20141488 (to Q.Y.), Preventive Medicine Foundation of Jiangsu Provincial Commission of Health and Family Planning Y2013058 (to Q.Y.), Jiangsu Provincial Distinguished Medical Experts Program Foundation (to Q.Y.), Jiangsu Provincial Innovation Team Program Foundation (to Q.Y.), Jiangsu Provincial Six talent Peaks Program Foundation (to Q.Y.) and Jiangsu Provincial 333 high level talents Program Foundation (to Q.Y.).

\section{REFERENCES}

1. Hanahan D, Weinberg RA. Hallmarks of cancer: the next generation. Cell. 2011; 144:646-674.

2. Kaelin WG, Jr. Cancer and altered metabolism: potential importance of hypoxia-inducible factor and 2-oxoglutaratedependent dioxygenases. Cold Spring Harb Symp Quant Biol. 2011; 76:335-345.

3. Sciacovelli M, Frezza C. Oncometabolites: Unconventional triggers of oncogenic signalling cascades. Free radical biology \& medicine. 2016 Nov; 100:175-181.

4. Hanahan D, Folkman J. Patterns and emerging mechanisms of the angiogenic switch during tumorigenesis. Cell. 1996; 86:353-364.

5. Song K, Wu H, Rahman HN, Dong Y, Wen A, Brophy ML, Wong S, Kwak S, Bielenberg DR, Chen H. Endothelial epsins as regulators and potential therapeutic targets of tumor angiogenesis. Cell Mol Life Sci. 2016 Aug 29.

6. Sonveaux P, Copetti T, De Saedeleer CJ, Vegran F, Verrax J, Kennedy KM, Moon EJ, Dhup S, Danhier P, Frerart F, Gallez B, Ribeiro A, Michiels C, Dewhirst MW, Feron O. Targeting the lactate transporter MCT1 in endothelial cells inhibits lactate-induced HIF-1 activation and tumor angiogenesis. PLoS One. 2012; 7:e33418.

7. Tretter L, Patocs A, Chinopoulos C. Succinate, an intermediate in metabolism, signal transduction, ROS, hypoxia, and tumorigenesis. Biochim Biophys Acta. 2016; 1857:1086-1101.

8. Kushnir MM, Komaromy-Hiller G, Shushan B, Urry FM, Roberts WL. Analysis of dicarboxylic acids by tandem mass spectrometry. High-throughput quantitative measurement of methylmalonic acid in serum, plasma, and urine. Clin Chem. 2001; 47:1993-2002.

9. Tannahill GM, Curtis AM, Adamik J, Palsson-McDermott EM, McGettrick AF, Goel G, Frezza C, Bernard NJ, Kelly B, Foley NH, Zheng L, Gardet A, Tong Z, et al. Succinate is an inflammatory signal that induces IL-1beta through HIF1alpha. Nature. 2013; 496:238-242.
10. He W, Miao FJ, Lin DC, Schwandner RT, Wang Z, Gao J, Chen JL, Tian H, Ling L. Citric acid cycle intermediates as ligands for orphan G-protein-coupled receptors. Nature. 2004; 429:188-193.

11. Koivunen P, Hirsila M, Remes AM, Hassinen IE, Kivirikko KI, Myllyharju J. Inhibition of hypoxia-inducible factor (HIF) hydroxylases by citric acid cycle intermediates: possible links between cell metabolism and stabilization of HIF. J Biol Chem. 2007; 282:4524-4532.

12. Selak MA, Armour SM, MacKenzie ED, Boulahbel H, Watson DG, Mansfield KD, Pan Y, Simon MC, Thompson $\mathrm{CB}$, Gottlieb E. Succinate links TCA cycle dysfunction to oncogenesis by inhibiting HIF-alpha prolyl hydroxylase. Cancer Cell. 2005; 7:77-85.

13. Mills E, O'Neill LA. Succinate: a metabolic signal in inflammation. Trends Cell Biol. 2014; 24:313-320.

14. Correa PR, Kruglov EA, Thompson M, Leite MF, Dranoff JA, Nathanson MH. Succinate is a paracrine signal for liver damage. J Hepatol. 2007; 47:262-269.

15. Sapieha P, Sirinyan M, Hamel D, Zaniolo K, Joyal JS, Cho JH, Honore JC, Kermorvant-Duchemin E, Varma DR, Tremblay S, Leduc M, Rihakova L, Hardy P, et al. The succinate receptor GPR91 in neurons has a major role in retinal angiogenesis. Nat Med. 2008; 14:1067-1076.

16. McCreath KJ, Espada S, Galvez BG, Benito M, de Molina A, Sepulveda P, Cervera AM. Targeted disruption of the SUCNR1 metabolic receptor leads to dichotomous effects on obesity. Diabetes. 2015; 64:1154-1167.

17. Rubic T, Lametschwandtner G, Jost S, Hinteregger S, Kund J, Carballido-Perrig N, Schwarzler C, Junt T, Voshol H, Meingassner JG, Mao X, Werner G, Rot A, Carballido JM. Triggering the succinate receptor GPR91 on dendritic cells enhances immunity. Nat Immunol. 2008; 9:1261-1269.

18. Robben JH, Fenton RA, Vargas SL, Schweer H, Peti-Peterdi J, Deen PM, Milligan G. Localization of the succinate receptor in the distal nephron and its signaling in polarized MDCK cells. Kidney Int. 2009; 76:1258-1267.

19. Yang L, Yu D, Fan HH, Feng Y, Hu L, Zhang WY, Zhou K, Mo XM. Triggering the succinate receptor GPR91 enhances pressure overload-induced right ventricular hypertrophy. Int J Clin Exp Pathol. 2014; 7:5415-5428.

20. Vargas SL, Toma I, Kang JJ, Meer EJ, Peti-Peterdi J. Activation of the succinate receptor GPR91 in macula densa cells causes renin release. J Am Soc Nephrol. 2009; 20:1002-1011.

21. Lamalice L, Le Boeuf F, Huot J. Endothelial cell migration during angiogenesis. Circ Res. 2007; 100:782-794.

22. Bayliss PE, Bellavance KL, Whitehead GG, Abrams JM, Aegerter S, Robbins HS, Cowan DB, Keating MT, O'Reilly T, Wood JM, Roberts TM, Chan J. Chemical modulation of receptor signaling inhibits regenerative angiogenesis in adult zebrafish. Nature chemical biology. 2006; 2:265-273. 
23. Mullen AR, DeBerardinis RJ. Genetically-defined metabolic reprogramming in cancer. Trends in endocrinology and metabolism: TEM. 2012; 23:552-559.

24. Yang M, Soga T, Pollard PJ. Oncometabolites: linking altered metabolism with cancer. The Journal of clinical investigation. 2013; 123:3652-3658.

25. McWhinney SR, Pasini B, Stratakis CA, International Carney T, Carney-Stratakis Syndrome C. Familial gastrointestinal stromal tumors and germ-line mutations. N Engl J Med. 2007; 357:1054-1056.

26. Ferrara N, Gerber HP, LeCouter J. The biology of VEGF and its receptors. Nat Med. 2003; 9:669-676.

27. Ehinger JK, Piel S, Ford R, Karlsson M, Sjovall F, Frostner EA, Morota S, Taylor RW, Turnbull DM, Cornell C, Moss SJ, Metzsch C, Hansson MJ, Fliri H, Elmer E. Cell-permeable succinate prodrugs bypass mitochondrial complex I deficiency. Nat Commun. 2016; 7:12317.

28. Rotstein OD, Nasmith PE, Grinstein S. pH-dependent impairment of the neutrophil respiratory burst by the Bacteroides byproduct succinate. Clin Invest Med. 1988; 11:259-265.

29. Mukhopadhyay D, Nagy JA, Manseau EJ, Dvorak HF. Vascular permeability factor/vascular endothelial growth factor-mediated signaling in mouse mesentery vascular endothelium. Cancer Res. 1998; 58:1278-1284.

30. Mavria G, Vercoulen Y, Yeo M, Paterson H, Karasarides M, Marais R, Bird D, Marshall CJ. ERK-MAPK signaling opposes Rho-kinase to promote endothelial cell survival and sprouting during angiogenesis. Cancer Cell. 2006; 9:33-44.

31. Hu J, Li T, Du S, Chen Y, Wang S, Xiong F, Wu Q. The MAPK signaling pathway mediates the GPR91-dependent release of VEGF from RGC-5 cells. Int J Mol Med. 2015; 36:130-138.

32. Chen Z, Han ZC. STAT3: a critical transcription activator in angiogenesis. Med Res Rev. 2008; 28:185-200.

33. Chung J, Uchida E, Grammer TC, Blenis J. STAT3 serine phosphorylation by ERK-dependent and -independent pathways negatively modulates its tyrosine phosphorylation. Molecular and cellular biology. 1997; 17:6508-6516.

34. Mu X, Shi W, Sun L, Li H, Jiang Z, Zhang L. Pristimerin, a triterpenoid, inhibits tumor angiogenesis by targeting VEGFR2 activation. Molecules. 2012; 17:6854-6868.

35. Wei J, Zhang J, Si Y, Kanada M, Zhang Z, Terakawa S, Watanabe H. Blockage of LMP1-modulated store-operated $\mathrm{Ca}(2+)$ entry reduces metastatic potential in nasopharyngeal carcinoma cell. Cancer Lett. 2015; 360:234-244. 